\title{
The Effectiveness and Tolerability of the High Dose Donepezil at 23 mg Tablet per Day for Alzheimer's Disease: A Meta-analysis of Randomized Controlled Trials
}

\author{
Adrian I. Espiritu ${ }^{1,2}$ and Alvin Rae F. Cenina ${ }^{1}$ \\ ${ }^{1}$ Department of Neurosciences, College of Medicine and Philippine General Hospital, University of the Philippines Manila \\ ${ }^{2}$ Department of Clinical Epidemiology, College of Medicine, University of the Philippines Manila
}

\begin{abstract}
Objective. To assess the effectiveness and tolerability of the $23 \mathrm{mg}$ tablet donepezil in patients with Alzheimer's disease (AD) using meta-analysis of randomized controlled trials.
\end{abstract}

Methods. Major healthcare databases were searched from May to September 2016. Evaluation of relevant trials, assessment of risk of bias, collection and analyses of data were performed.

Results. A total of 1,774 adult participants with $A D$ were pooled from the two trials included. Pooled data showed that after 24 weeks of treatment, no significant difference was noted between Donepezil $23 \mathrm{mg} /$ day and Donepezil $10 \mathrm{mg} /$ day in terms of cognitive function (1.06 SIB points [-0.13, 2.26]; 1704 participants) and in terms of global clinical assessment $(-0.02 \mathrm{CIBIC}+$ points [-0.13, 0.09]; 1705 participants). The participants who took the higher dose were at higher risk to experience "any adverse event" than those who received the lower dose (1.17 RR [1.09, 1.26]; 1785 participants).

Conclusion. Current evidences do not support the routine use of Donepezil $23 \mathrm{mg}$ tablet for the improvement of cognitive function and global clinical status of patients with AD. The higher dose is also marked with an increased incidence of adverse events compared to the lower dose.

Key Words: high dose Donepezil, Donepezil 23 mg, Alzheimer's Disease, meta-analysis

This study was published in abstract form in the Journal of the Neurological Sciences last October 2017.

Poster Presentation, XXIII World Congress of Neurology (WCN 2017), September 16-21, 2017, Kyoto International Conference Center, Kyoto, Japan.

Podium Presentation, Second Place Winner, Meta-analysis Category, $38^{\text {th }}$ Philippine Neurological Association Annual Convention, November 16 to 19, 2016, Novotel Manila Araneta Center, Quezon City, Philippines.

Podium Presentation, Second Place Winner, Meta-analysis Category, Philippine General Hospital (PGH) Residents' Research Forum, November 8, 2016, Human Resource Development Division Conference Room, Philippine General Hospital, Taft Avenue, Manila, Philippines.

Podium Presentation, Third Place Winner, Meta-analysis Category, PGH Department of Neurosciences Research Forum, October 5, 2016, Alvior Hall, University of the Philippines College of Medicine, Pedro Gil Street, Ermita, Manila, Philippines.

Corresponding author: Adrian I. Espiritu, MD

Department of Neurosciences

Philippine General Hospital

University of the Philippines Manila

Taft Avenue, Manila 1000, Philippines

Email: espirituadrian@gmail.com 


\section{INTRODUCTION}

It is estimated that in every 3.2 seconds, a new case of dementia emerges, yielding an anticipated figure of 9.9 . million new cases annually., Alzheimer's disease (AD), the most common form of dementia, is a neurodegenerative disorder which manifests in the older population which initially presents with gradually progressive cognitive decline and subsequent deterioration in executive dysfunction, visuospatial skills, language skills, and behavioral changes.

In patients with $\mathrm{AD}$, acetylcholine production is diminished from impaired synthesis of choline acetyltransferase. It was hypothesized that enhancing cholinergic activity in individuals with $\mathrm{AD}$ would have a beneficial response. ${ }^{3}$ Cholinesterase inhibitors, such as donepezil, which prevents the breakdown of acetylcholine by interfering with the action of acetylcholinesterase at the synaptic cleft, was found to have modest benefits in patients with $\mathrm{AD}$ and have tolerable safety profile. ${ }^{4}$ Donepezil is administered once a day, given at $5 \mathrm{mg}$ per day for 4 weeks, then typically shifted to a $10 \mathrm{mg}$ per day thereafter for possible further clinical benefits. ${ }^{5}$

In 2010, a higher dose formulation of donepezil at 23 mg was introduced. ${ }^{6}$ The rationale for the development of this higher dose were the following: the use of $5 \mathrm{mg}$ and $10 \mathrm{mg}$ of donepezil inhibited cortical anticholinesterase activity in vivo by only $20-40 \%$ as shown in modern imaging analyses, indicating that a higher dose may offer better efficacy; brain tissues collected from patients with $\mathrm{AD}$ with more advanced symptoms have greater cholinergic deficits, suggesting that a higher dose acetylcholinesterase inhibitors may be warranted for this population; there was a note of dose-response relationship wherein patients with advanced $\mathrm{AD}$ who took the $10 \mathrm{mg}$ dose per day received more beneficial outcomes; and a study employing 20 $\mathrm{mg}$ donepezil per day appears to be safe as reported in a small pilot randomized controlled trial involving mild to moderate $\mathrm{AD}$ who already tolerated $10 \mathrm{mg}$ per day donepezil dose initially. 7,8 The higher dose has a maximum plasma concentration $\left(\mathrm{C}_{\max }\right)$ and time to $\mathrm{C}_{\max }$ which are nearly twice as great compared to $10 \mathrm{mg}$ dose. ${ }^{7}$ The $23 \mathrm{mg}$ dose can also sustain a higher blood levels which may lengthen efficacy and has a slower rise to $\mathrm{C}_{\max }$ which can decrease incidence of adverse events compared to the standard dose. ${ }^{7}$ Consequently, these pharmacological and pharmacokinetic properties of the higher dose may theoretically improve and prolong effectiveness and possibly diminish the harmful effects in patients with $\mathrm{AD} .^{7}$

This review aims to pool the evidences on the effectiveness and tolerability of the $23 \mathrm{mg}$ dose donepezil in patients with $\mathrm{AD}$ by using meta-analysis of available randomized controlled trials employing the rigorous guidelines indicated in the Cocbrane Handbook for Systematic Reviews of Interventions and in the PRISMA (Preferred Reporting Items for Systematic revierws and Meta-analyses) Statement. ${ }^{9,10}$

\section{METHODS}

Trials which employed randomized, at least doubleblind, placebo- and/or active-controlled designs were included in this review. Studies utilizing other designs, such as quasi-experimental (nonrandomized controlled design and pre/posttest design), cluster-randomized, cross-over, prospective/retrospective cohort, case-control and crosssectional trials, were not considered for this review.

Trials which included adults, male or female, with a diagnosis of Alzheimer's disease that generally conforms with the National Institute of Neurological and Communicative Diseases and Stroke-Alzheimer's Disease and Related Disorders Association criteria (NINCDSADRDA; updated as the National Institute on Aging and the Alzheimer's Association (NIA-AA) criteria) or with the Diagnostic and Statistical Manual for Mental Disorders (DSM-IV and DSM-5) criteria were included in this review. ${ }^{11,12}$

No restrictions were implemented in terms of race, economic standing, disease severity, comorbidities and previous use of other anticholinesterase inhibitors and/or concurrent use of memantine. Studies involving participants with other causes of dementia were excluded. Studies employing the intervention Donepezil $23 \mathrm{mg}$ daily dose regimen for the symptomatic treatment of $\mathrm{AD}$ compared to placebo and/or any active agent/s were included.

The following databases were searched in May to September 2016 for relevant articles: The Cochrane Central Register for Controlled Trials (CENTRAL) by The Cochrane Library, MEDLINE by PubMed, Embase, Scopus, LILACS (Literatura Latino-Americana e do Caribe em Ciências da Saúde), and ClinicalTrials.gov website. The general search and corresponding $\mathrm{MeSH}$ or Emtree terms that were used include the following: (donepezil OR Aricept OR "E 2020" OR E-2020 OR E2020) AND ("high dose" OR high-dose OR "23 mg”) AND (alzheimer* disease OR alzheimer* dementia). Other available databases including The HERDIN Database of the Philippines, websites of the Philippine Neurological Association and the Philippine College of Physicians, and other local medical journals were searched for other relevant trials. Reference lists of relevant articles (retrieved studies and review articles) were explored for other possible significant trials.

Screening criteria was applied using the titles and abstracts of all possible relevant trials. Full-text articles were retrieved if the information was insufficient to answer any of the screening criteria. Relevant trials, which passed the screening criteria, were retrieved in full text articles and were reviewed using the eligibility criteria. The trials which fulfilled the eligibility criteria were included in this review.

Trials which reported secondary data (i.e., data extracted from other trials) and in which no full-text report was accessible were excluded. Any duplicate reports of the same study, post hoc analyses, abstract-only reports, results with no 
accompanying described background and methods, reviews, and animal studies were discarded from the review.

The data collected from the included trials were as follows: the methods or study design, the participants with the pertinent inclusion and exclusion criteria, trial settings, the details of the treatment given to the intervention and the control groups such as dosage, route, duration, preparation and formulation, and all the relevant outcomes measured in the study. Both continuous and dichotomous data were extracted from the included studies. For the continuous outcomes, mean differences, standard errors of the mean changes, and the total participants in all the treatment arms were obtained. Standard deviations of mean changes were derived from the available data. For dichotomous outcomes, the number of participants who experienced the outcome of interest and the total number of participants in each arm were collected.

Risk of bias evaluation of the included studies were performed. The Cochrane Collaboration's tool for assessment of risk of bias were employed for this review. ${ }^{9}$

For the primary outcomes, missing data in the "observed case" analyses from the included studies were substituted for last observation carried forward (LOCF).

For continuous data, the effect measures were mean differences with $95 \%$ confidence intervals $(\mathrm{CI})$. On the other hand, for dichotomous data, risk ratios with 95\% CI were employed.

Clinical heterogeneity was assessed by comparing the population, intervention, comparison and outcome measures of the included studies. Methodological heterogeneity was evaluated by comparing the study designs and risk of bias in the trials. Statistical heterogeneity was detected using the chi-squared $\left(\mathrm{X}^{2}\right)$ test with a $\mathrm{p}$-value $<0.10$ to indicate statistically significant heterogeneity. The degree of heterogeneity was measured using $\mathrm{I}^{2}$ statistic with values 0 to $40 \%$ indicating unimportant statistical heterogeneity. ${ }^{9}$ The collected data were synthesized and analyzed using the Review Manager 5.3 (RevMan 5.3) (Copenhagen: The Nordic Cochrane Centre, The Cochrane Collaboration,2012).

Using the fixed effects model, meta-analyses were performed employing the inverse-variance method for the continuous outcomes, and Cochran-Mantel-Haenszel method for the dichotomous data. A statistically significant difference between the intervention and the control arms was noted if the $95 \%$ CI of the mean differences did not include the number zero for the continuous outcomes, and if the $95 \% \mathrm{CI}$ of the relative risks did not include the number one for the dichotomous outcomes.

\section{RESULTS}

There were 6 major databases that were explored for possible relevant articles. The search generated 207 records using the formulated database-specific search strategies. Duplicate reports (75 records) were discarded. Screening criteria were applied to the remaining 132 records and from these group, 104 articles were excluded based on the published title and abstract. The number of articles assessed for eligibility were 28. After applying the formulated eligibility criteria, only 2 studies met the inclusion criteria for this review. Figure 1 summarizes the flow diagram of information of the review.

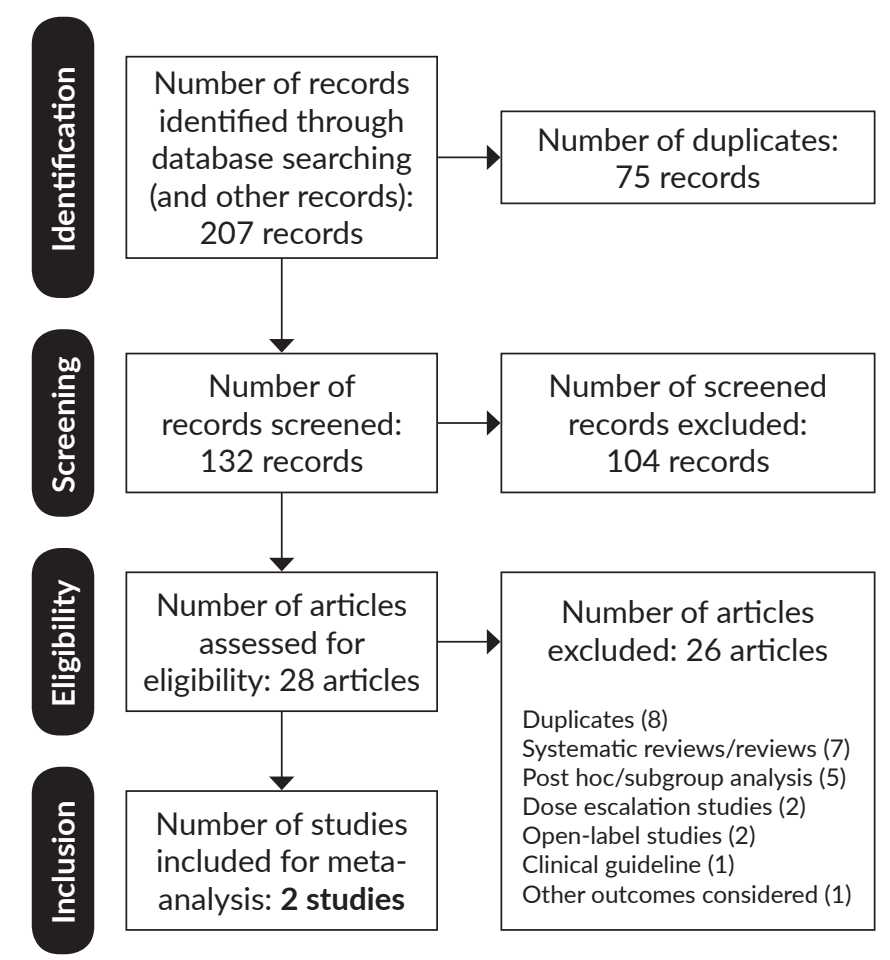

Figure 1. Flow diagram of information of the review.

The two trials included were Farlow 2010 and Homma $2016{ }^{6,13}$ The trials aimed to determine the effectiveness and tolerability of the higher donepezil dose $(23 \mathrm{mg} /$ day) compared to the standard, immediate-release dose of donepezil (10 mg/day). These trials were previously registered in the ClinicalTrials.gov website, with identifiers NCT00478205 and NCT01539031, respectively.

Both trials employed multicenter, randomized, double-blind, parallel-group methodological designs. The intervention $(23 \mathrm{mg} /$ day $)$ and comparator $(10 \mathrm{mg} /$ day $)$ of interest were similar in both studies. Primary outcomes for both studies were similar, which was cognitive function, as measured by Severe Impairment Battery (SIB) change from baseline score, and global clinical changes from baseline, as quantified by Clinician's Interview-Based Impression of Change Plus Caregiver Input (CIBIC+) change from baseline. Both trials reported adverse events associated with the treatment arms. Other outcomes measured, such as the Mini-Mental State Examination (MMSE) and the Alzheimer's Disease Cooperative Study-Activities of Daily Living (ADCS-ADL), were reported in one study. ${ }^{6}$ 
A total of 1,774 participants (PPA) were pooled from the two trials included. The Farlow 2010 study employed DSM-IV and NINCDS-ADRDA criteria to diagnose probable $\mathrm{AD}$ and enrolled moderate to severe cases (MMSE scores 0-20), while the Homma 2016 trial used the DSM-IV-TR and registered only the severe ones (MMSE scores 1-12).

Mean ages ranged from 73.8 to 76 and most individuals were female (1,137 participants; 64.1\%). Farlow 2010 trial was a multiracial trial consisted mostly of whites (1,054 participants; 73.5\%), asians/pacific islanders (248 participants; 17.3\%), hispanics (93 participants; 6.5\%), and blacks (31 participants; 2.2\%). On the other hand, Homma 2016 trial included only Japanese patients. Relatively more individuals with increased weights were enrolled in Farlow 2010 ( $\geq 55 \mathrm{~kg}: 1,104$ participants [77\%]) while the other study registered slightly increased number of patients with heavier weights in Homma 2016 ( $\geq 55$ kg; 192 participants [56.5\%]). All participants in both studies required at least 3-month duration of intake of Donepezil $10 \mathrm{mg}$ per day prior to screening. Both studies permitted the concomitant use of memantine. Farlow 2010 specified that the other anticholinergic medications must be halted for at least 3 months prior to screening while in Homma 2016, no such report was made in the published article. Other comorbidities needed to be clinically stable in both trials.

Both studies employed computer-generated randomization codes differing only randomization ratios: 1:1 (Homma 2016), 2:1 (Farlow 2010). However, in terms of allocation sequence concealment, no detailed method was reported in both trials; thus, both studies received an "unclear" risk for this domain. Double-blinding was done in both studies; thus, blinding of both the participants/ caregivers and investigators/study personnel/outcome assessors were assumed. To maintain masking of treatment arms, both trials employed a double dummy design; however, only Homma 2016 provided sufficient information on the manner it was performed. Attrition rate in both trials for the main outcomes were considered significant: 26.1\% (Farlow 2010) and 21.9\% (Homma 2016); hence both trials were considered "high risk" for attrition bias. To impute missing data in both trials, the "Last Observation Carried Forward" (LOCF) method was used. No published protocols were identified through database searching; however, both trials were registered in ClinicalTrials.gov website, as follows: NCT00478205 (Farlow 2010) and NCT01539031 (Homma 2016). Other probably significant outcomes for drug trials for $\mathrm{AD}$ were not measured in the included studies (e.g., behavioral disturbance, quality of life, effects on carer, dependency, acceptability of treatment, and cost); hence, both trials received "unclear risk" for reporting bias.

One comparison (Donepezil $23 \mathrm{mg} /$ day vs. Donepezil $10 \mathrm{mg} /$ day) was generated from the data presented in the 2 included studies (Farlow 2010; Homma 2016). The intervention of interest, Donepezil $23 \mathrm{mg} /$ day, was not compared to placebo or to any other active agent for Alzheimer's Disease.

Cognitive function (Severe Impairment Battery, SIB)

Both studies (Farlow 2010; Homma 2016) measured cognitive function using SIB, in terms of Least Squares Mean Difference (LSMD) between the baseline score and the score after 24 weeks. Missing data was imputed using "Last Observation Carried Forward" (LOCF). After 24 weeks, no significant difference was noted between Donepezil $23 \mathrm{mg} /$ day and Donepezil $10 \mathrm{mg} /$ day when all participants were included in the analysis (1.06 SIB points [-0.13, 2.26]; 1,704 participants [2 studies; Farlow 2010; Homma 2016]). There was substantial statistical heterogeneity between the two studies $\left(\mathrm{I}^{2}=69 \%\right)$. The forest plot for this outcome is shown in Figure 2.

\section{Cognitive Function (Mini-Mental State Examination, MMSE)}

Only one trial (Farlow 2010) reported data on MMSE as a cognitive outcome measure presented as LSMD between the baseline score and the score after 24 weeks. LOCF was employed to fill in the missing data. No significant difference was noted between the treatment arms when all participants were included (0.20 MD [-0.33, 0.73); 1,370 participants [1 study; Farlow 2010].

\section{Global Clinical Assessment}

The two studies (Farlow 2010; Homma 2016) contributed relevant data for global clinical assessment, as rated by Clinician's Interview-Based Impression of Change Plus Caregiver Input (CIBIC+), employing Least Squares Mean (LSM) as measure of effect. Missing data was imputed using LOCF. After 24 weeks, there was no significant difference seen between Donepezil $23 \mathrm{mg} /$ day and Donepezil $10 \mathrm{mg} /$ day when all participants were integrated in the overall estimate $(-0.02$ CIBIC+ points [-0.13, 0.09]; 1705 participants (2 studies; Farlow 2010 and Homma 2016]). Statistical heterogeneity was significant $\left(\mathrm{I}^{2}=65 \%\right)$. The forest plot for this outcome is shown in Figure 2.

\section{Activities of Daily Living (Alzheimer's Disease Cooperative Study - Activities of Daily Living (severe version), ADCS-ADL-sev)}

One study (Farlow 2010) provided data on the outcome ADCS-ADL-sev using LSMD between the baseline score and the score after 24 weeks. LOCF was used to impute for missing data. After 24 weeks, no significant difference between the treatment arms was noted when all the individuals were included in the analysis (0.00 MD [-1.18, 1.18]; 1369 participants [1 study; Farlow 2010]).

\section{Adverse events}

Both studies (Farlow 2010; Homma 2016) provided relevant data for adverse events. Significantly more adverse 


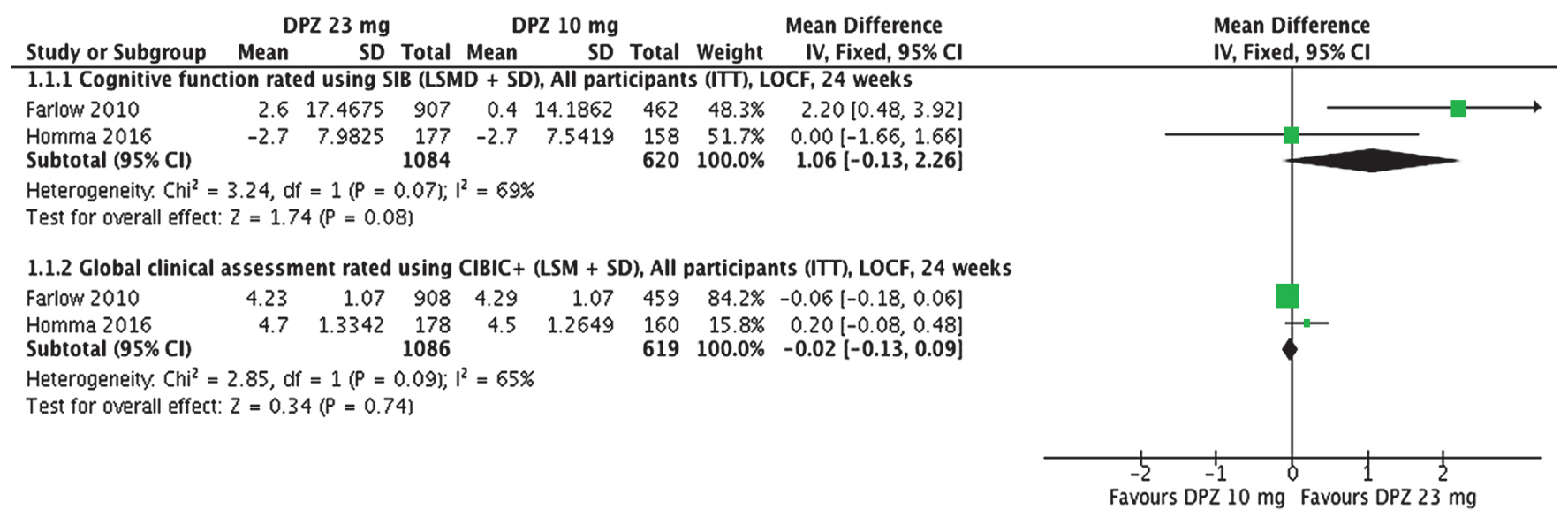

Figure 2. Forest plot of comparison "Donepezil $23 \mathrm{mg} /$ day vs Donepezil $10 \mathrm{mg} /$ day" for cognitive function as rated by SIB, Severe Impairment Battery, and for global clinical assessment as rated by CIBIC+, Clinician's Interview-Based Impression of Change Plus Caregiver Input.

${ }^{*} \mathrm{Chi}^{2}\left(\mathrm{X}^{2}\right)$, a test to detect statistical heterogeneity; $\mathrm{Cl}$, Confidence Interval; df, degrees of freedom; DPZ, Donepezil; Fixed, Fixed-effects model; ITT, Intention-To-Treat; I ${ }^{2}$, a test to determine degree of statistical heterogeneity; IV, Inverse variance method; LOCF, "Last Observation Carried Forward"; LSM, Least Squares Mean; LSMD, Least Squares Mean Difference; P, p-value; SD, Standard Deviation; Z, standard score.

events occurred in the "Donepezil $23 \mathrm{mg} /$ day" arm than "Donepezil $10 \mathrm{mg} /$ day" arm for "any adverse event" (1.17 RR [1.09, 1.26]; 1,785 participants [2 studies; Farlow 2010 and Homma 2016]). Furthermore, it was found out that intake of Donepezil $23 \mathrm{mg} /$ day was associated with increased risk for the following specific adverse events compared to Donepezil $10 \mathrm{mg} /$ day, as follows: a) anorexia/decreased appetite (2.79 RR [1.59, 4.87]; 1785 participants [2 studies: Farlow 2010 and Homma 2016]); b) bradycardia and sinus bradycardia (4.40 RR [1.34, 14.44]; 1434 participants [1 study: Farlow 2010]); c) contusion (9.78 RR [1.32, 72.67]; 1434 participants [1 study: Farlow 2010]); d) diarrhea (1.69 RR [1.14, 2.52]; 1785 participants [2 studies: Farlow 2010 and Homma 2016]); e) nausea (3.12 RR [1.97, 4.94]; 1785 participants [2 studies: Farlow 2010 and Homma 2016]); f) vomiting (3.62 RR [2.15, 6.09]; 1785 participants [2 studies: Farlow 2010 and Homma 2016]); and g) weight decreased (1.99 RR [1.11, 3.54]; 1785 participants [2 studies: Farlow 2010 and Homma 2016]).

The forest plots of the adverse events are presented in Figure 3. Serious adverse events between the treatment arms were not significantly different for "any serious adverse event" i.e., aggression, confusional state, death, fall, pneumonia, urinary tract infection, and syncope.

\section{DISCUSSION}

The overall evaluation of evidences on the effectiveness and tolerability of an intervention requires the scrutiny of the properties of the drug itself, the suitability of the methodology performed in the trials, the characteristics of the target population, and the appropriateness of the outcomes measured. This review included two studies
(Farlow 2010 and Homma 2016) which uniformly evaluated the effectiveness of donepezil at $23 \mathrm{mg}$ per tablet administered once a day.

The designs employed in the studies were randomized, controlled, parallel group trials. Both trials were performed in multiple centers. The first published study, Farlow 2010, was completed in 219 sites in Asia, Europe, Australia, North America, South Africa, and South America (majority were from the white race). The second trial, Homma 2016, was implemented in 69 sites in Japan.

The target population of the studies were individuals with probable Alzheimer's Disease (AD), diagnosed using stringent, well-accepted similar criteria, differing only in severity. Farlow 2010 trial registered participants with moderate-severe disease while Homma 2016 study admitted individuals with the most severe cases. Both studies enrolled $\mathrm{AD}$ patients with the following important key characteristics: must be on immediate-release donepezil at $10 \mathrm{mg}$ per tablet once a day for at least 3 months prior to administration of the $23 \mathrm{mg}$ sustained-release dose; must have discontinued intake of other drugs in the cholinesterase inhibitors class; must have stable comorbidities; and may be enrolled regardless of concurrent intake of memantine.

Both trials employed several outcome measures considered standard in the drug trials for AD. The studies measured cognitive function (rated using the Severe Impairment Battery) and global clinical assessment (rated using the Clinician's Interview-Based Impression of Change Plus Caregiver Input, $\mathrm{CIBIC+)}$ as co-primary outcome measures. Other cognitive scale, the Mini-Mental State Examination (MMSE), was also evaluated in Farlow 2010. Additional secondary outcomes were activities of daily living (measured using the Alzheimer's Disease Cooperative 


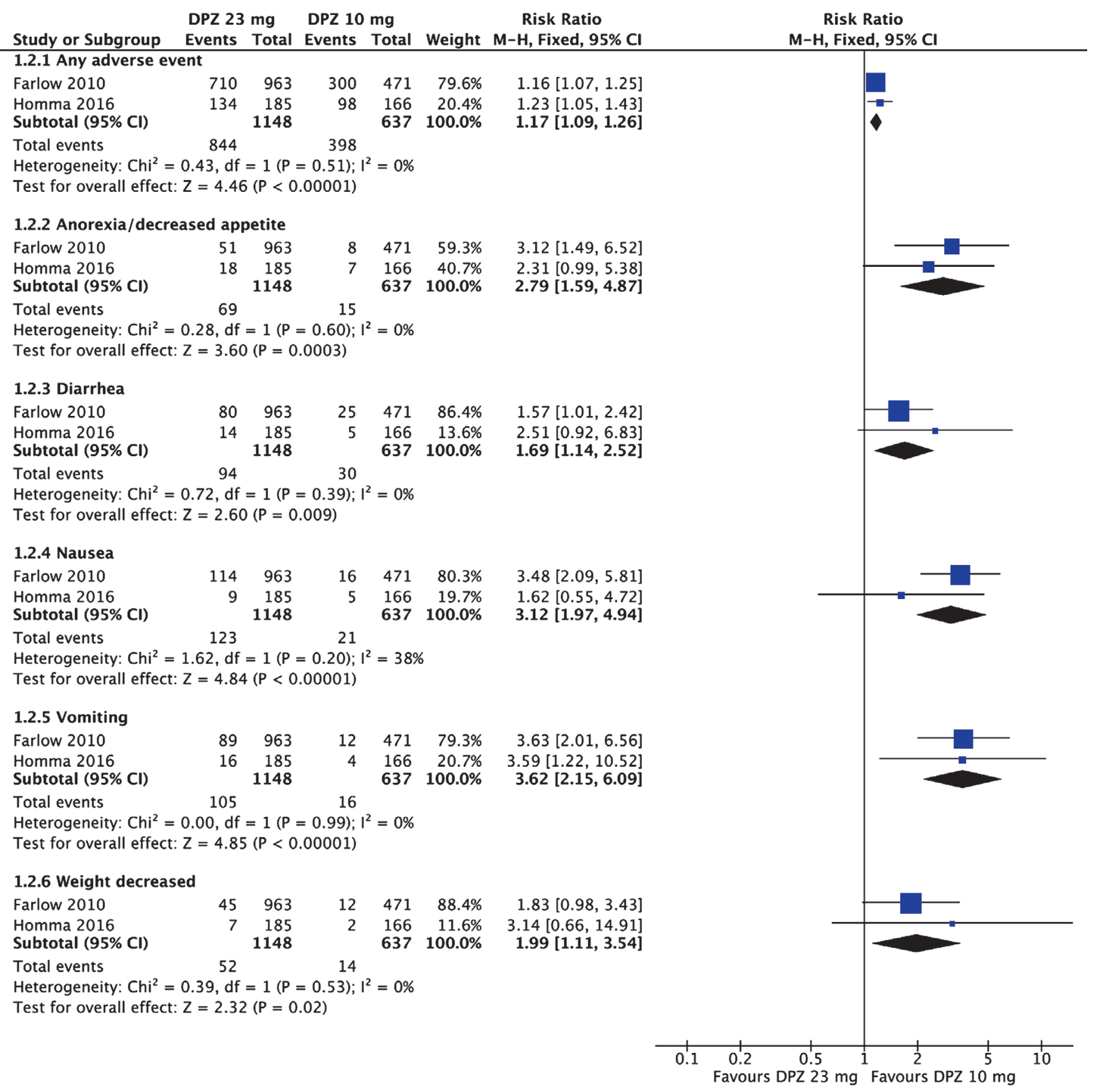

Figure 3. Forest plot of comparison "Donepezil $23 \mathrm{mg} /$ day vs Donepezil $10 \mathrm{mg} / \mathrm{day}$ " for the adverse events showing significant difference between the treatment groups.

${ }^{*} \mathrm{Chi}^{2}\left(\mathrm{X}^{2}\right)$, a test to detect statistical heterogeneity; $\mathrm{Cl}$, Confidence Interval; df, degrees of freedom; DPZ, Donepezil; Fixed, Fixedeffects model; $I^{2}$, a test to determine degree of statistical heterogeneity; $M-H$, Mantel-Haenszel method, $P, p$-value; $Z$, standard score.

Study - Activities of Daily Living scale (severe version), ADCS-ADL-sev), as reported in Farlow 2010 and adverse events associated with the drug administered, as reported by the two studies. All treatment effects were measured at 24-week duration.

The pooled analysis of the two studies suggested that Donepezil $23 \mathrm{mg} /$ day for the symptomatic treatment of
AD was not significantly different from Donepezil $10 \mathrm{mg} /$ day in terms of cognitive function and global assessment of change when all populations were included in the analysis at the end of the 24-week treatment. This is supported by the insignificant changes in SIB (1.06 SIB points $[-0.13,2.26])$ and CIBIC+ points $(-0.02$ CIBIC+ points $[-0.13,0.09])$ between the higher and lower donepezil doses mentioned. 
For the cognitive function in terms of SIB, a substantial heterogeneity can be appreciated $\left(\mathrm{I}^{2}=69 \%\right)$. Subgroup analysis of Farlow 2010 showed that the higher dose donepezil was favorable for patients with more advanced $\mathrm{AD}$ (MMSE 0-16) (3.1 SIB points effect size). In the subgroup of US participants, which represents the largest subpopulation in the Farlow 2010 trial and who were considered more cognitively impaired at the start of the trial, compared to the overall population, exhibited significant difference in favor of the higher donepezil dose (3.9 SIB point effect size). ${ }^{7}$ It is interesting to note that in Homma 2016 trial, which enrolled and randomized more severe AD patients (MMSE 1-12), did not show the same statistical beneficial effect favoring the higher dose donepezil in terms of cognitive function using SIB. Although the subgroup analysis displayed a beneficial cognitive outcome in Farlow 2010, this should be interpreted with caution because comparisons made in subgroup analysis are observational in nature and suffers from the lack of randomization. ${ }^{9}$ The effects seen on cognitive function as rated by SIB in Homer 2016 may be less biased than the "more advanced AD" subgroup analysis in Farlow 2010. Additional confounding study-level characteristic might explain the observed difference in the subgroup analyses described.

In terms of global clinical changes, the results of the two studies also displayed substantial heterogeneous results $\left(\mathrm{I}^{2}=65 \%\right)$. However, the reported subgroup analyses of subpopulations in Farlow 2010 including patients with/ without concurrent memantine use, patients more/less advanced AD, US and non-US participants, did not reveal statistical differences between the two treatment arms for this outcome (data not shown).

Incidence rates of various adverse events (AE) were found to be greater in patients who received Donepezil $23 \mathrm{mg} /$ day compared to those who took the Donepezil 10 $\mathrm{mg} /$ day. Majority of specific AEs, such as nausea, vomiting, diarrhea, were frequent due to its intrinsic cholinergic activity in the gastrointestinal system. ${ }^{14}$ Greater incidence of "bradycardia and sinus bradycardia" was also seen in the higher donepezil dose compared to the standard dose. This may be due to the activation of the muscarinic receptors in the pacemaker cells via the release of acetylcholine neurotransmitter from the vagal fibers. Thus, it is advisable to refrain prescribing the higher dose donepezil in patients with sick sinus syndrome, at risk for bradycardia or heart block, and those taking drugs known to reduce the heart rate (e.g. beta-blockers and digoxin). ${ }^{14} \mathrm{~A}$ cardiac evaluation, which may include a baseline electrocardiography and/or echocardiography that may be essential prior to the use of this regimen. Anorexia or decreased appetite and weight loss are more frequent in the $23 \mathrm{mg}$ dose than the $10 \mathrm{mg}$ dose. It is uncertain if disease progression of $\mathrm{AD}$ may contribute to their incidence. Lastly, reports of contusion is more common in the higher dose than the lower dose. The reason for this increased incidence is unclear.
Both trials were concluded after 24-week period of treatment. This period was considered arbitrary and treatment effects may have varied if they were measured for prolonged periods. A 24-week duration of treatment is relatively short if the life expectancy of $\mathrm{AD}$ patients is taken into account. Average life expectancy of patients with $\mathrm{AD}$ ranges from 3 to 10 years and the disease progresses very slowly. Therefore, an ideal drug trial duration involving this population would take years of study. ${ }^{15}$ However, conducting a trial for longer periods of time is intrinsically costly and investigators would probably settle at completing trials that would be sufficient for possible drug licensing purposes.

The Farlow 2010 employed a higher ratio for randomization (2:1) in which the "Donepezil $23 \mathrm{mg} /$ day" received around twice the number of participants compared to the "Donepezil $10 \mathrm{mg} /$ day" arm. The reason for this design was not stated in the published report. In this randomization scheme, the participants were likely believed to be receiving the higher dose donepezil than the lower dose.

Unequal randomization is usually performed to reduce the treatments cost, to avoid the loss of power from dropout or cross-over, ethics, and the gaining of additional information on the treatment. ${ }^{16}$ It was presumed in this review that higher randomization ratio was employed so that power to detect statistical difference is maintained since a substantial increase in drop-outs, likely due to adverse events, is expected if dose of donepezil were increased, as seen in other drug trials of the standard doses of donepezil. ${ }^{17}$

The effectiveness data for the included studies suffers greatly from increased attrition rates $(26.1 \%$ for Farlow 2010 and 21.9\% for Homma 2016). In order to attempt to analyze the data in an intention-to-treat manner, the large drop-outs of participants from the trials before study completion was compensated by the use of "Last Observation Carried Forward" (LOCF), to estimate for the missing data. This technique assumes that the patient's state at the time of dropout to the study completion is constant and disregards if there are improvements or deterioration of patient's condition in the same time period. Although this has become the standard for controlled trials even in dementia trials, employing LOCF in the analysis can potentially introduce bias and probably overestimate the effectiveness of the treatment of interest. This is especially true if values are not completely "missing at random" and may be due to drug adverse effects, symptoms or disease severity. ${ }^{18}$

Improvements in cognition is important in $\mathrm{AD}$, but this outcome did not automatically translate into observable clinical improvement, as compared to outcomes such as global clinical assessment or performance activities of daily living. Moreover, it appeared that very few significant outcomes were considered in these trials and other noteworthy outcomes were not measured in a drug trial for AD such as changes in global disease severity, behavioral disturbance, quality of life, effects on carer, dependency, acceptability of treatment, as well as the cost of treatment. High attrition 
rates were a major concern for the two included trials because effect measures may be affected by the systematic error particularly if LOCF method was employed.

The effects of Donepezil $23 \mathrm{mg} /$ day compared to Donepezil $10 \mathrm{mg} /$ day was evaluated in patients with moderate to severe AD, using the well-accepted criteria (DSMIV, DSM-IV-TR, and NINCDS-ADRDA) to clinically diagnose AD and using MMSE to grade the severity. Thus, the provided evidence in this review will specifically apply to this spectrum of $\mathrm{AD}$ severity. Data is not yet available for patients with mild severity of the disease at the time of search period of this review. In addition, the treatment arms were applied to participants with "stable comorbidities". This implied that the set of participants enrolled in these studies were considered "healthy individuals". Since the incidence of $\mathrm{AD}$ is highly distributed in the older age group who generally have multiple, possibly uncontrolled comorbidities and taking several drugs per day which could possibly affect the pharmacodynamic and pharmacokinetic properties of donepezil, the applicability of the results and the tolerability of drugs in this subpopulation will likely vary. Moreover, the relevant data was obtained in $\mathrm{AD}$ patients who took at least 3 months of Donepezil $10 \mathrm{mg}$ tablet once a day and did not take any other anticholinesterase inhibitors and regardless of concurrent memantine use. Therefore, the results of this review encompass this particular population.

All included trials employed a process of randomization but did not indicate a method of allocation concealment that was done in the trials. The baseline characteristics of the participants belonging to the treatment arms appeared to be similar; and thus, selection bias might have been prevented. The trials employed a double-blind study design to avoid performance and detection biases. To ensure avoidance of performance bias, a double dummy design of administering the intervention and placebo was done. For the prevention of detection bias, blinding of outcome assessors was presumed since the published reports indicated that "double-blind" was performed. Double-blinding in literature may mean blinding of the participants and the primary investigators while no attempts to blind the outcome assessors was done. In other trials, the indication of "double-blinding" in the methodology assumes that masking of the outcome assessors was also performed apart from the participants and the investigator. In this particular review, blinding of outcome assessors was deemed significant because the outcomes reported in the trials, although described using standardized well-accepted scales, may be subjective in nature and the information obtained were mostly patientor caregiver-reported data. Moreover, attrition rate for both studies was considered substantial and the employment of LOCF as a means to estimate data for dropped-out patients may introduce significant bias to the pooled treatment effects. For trials with high drop-out rates wherein adverse events are a clinical issue, attrition bias might significantly overestimate the effects of the intervention of interest.
The Expert Working Group (EWG) assembled last June 2011 to critically appraise the data on the large multinational trial on the effects of Donepezil $23 \mathrm{mg} /$ day on the cognitive function (rated using SIB) and global clinical assessment (rated using $\mathrm{CIBIC+}$ ) in patients with moderate to severe Alzheimer's disease (AD).6,7 The group integrated the available information and provided an extensive guideline on the application of this higher dose in the clinical setting. Based on the significant effects on the cognitive function, but not in the global clinical assessment when all participants were included in the analysis, the EWG concluded that Donepezil $23 \mathrm{mg}$ tablet given once a day is efficacious for patients with moderate to severe $\mathrm{AD}$ who needs additional favorable outcomes beyond the $10 \mathrm{mg}$ dose. The higher dose is only given if no further beneficial outcomes is appreciated in the $10 \mathrm{mg}$ dose and if the patient can tolerate the 10 $\mathrm{mg}$ dose prior to starting the $23 \mathrm{mg}$ dose. However, when the Japanese trial (Homma 2016) was released last January 2016 on the effects of Donepezil $23 \mathrm{mg} /$ day compared to Donepezil $10 \mathrm{mg} /$ day for patients with severe $\mathrm{AD}$, no significant difference was noted in terms of cognitive function and global clinical assessment rated using the same scales. When the treatment effects were pooled in this review, all the mentioned primary outcomes did not show significant differences between the mentioned treatment arms.

Further research should aim to develop a higher donepezil formulation which will clinically increase the effectiveness of the drug while minimizes the associated adverse events. Although cognitive function may be considered essential in every drug trial for $\mathrm{AD}$, more clinically significant outcomes such as global clinical assessment, changes in global disease severity, performance of daily living, behavioral disturbance, quality of life, effects on carer, and costs of the treatment. It is also suggested that the duration of future researches will be greater than 24 weeks to assess the intervention effects, either favorable or harmful, at lengthier periods of time.

\section{CONCLUSION}

This review synthesized the data on the effectiveness and tolerability of the $23 \mathrm{mg}$ donepezil dose compared to the standard $10 \mathrm{mg}$ donepezil dose. Pooled data showed that the $23 \mathrm{mg}$ dose of donepezil for the improvement of cognitive function and global clinical status of patients with moderate to severe $\mathrm{AD}$ did not show statistical significance when compared to the $10 \mathrm{mg}$ donepezil standard dose. The higher dose is also marked with an increased incidence of adverse events which may be considered a major clinical issue. Therefore, current evidences do not support the routine use of the $23 \mathrm{mg}$ donepezil in patients with $\mathrm{AD}$.

\section{Statement of Authorship}

AIE and ARFC contributed to the study concept, design of the work, data collection and analyses, critical writing and revision of the manuscript for intellectual content. 


\section{Author Disclosure}

All authors declared no conflicts of interest.

\section{Funding Source}

This research did not receive grant from any funding agency.

\section{REFERENCES}

1. Alzheimer's Disease International. The global impact of dementia: an analysis of prevalence, incidence, cost and trends [Internet]. [cited 2016 Aug]. Available from: https://www.alz.co.uk/research/ WorldAlzheimerReport2015.pdf.

2. Alzheimer's Association. Alzheimer's disease facts and figures [Internet]. 2016 [cited 2016 Aug]. Available from: http://www.alz.org/ documents_custom/2016-facts-and-figures.pdf.

3. Ferreira-Vieira TH, Guimaraes IM, Silva FF, Ribeiro FM. Alzheimer's disease: Targeting the Cholinergic System. Curr Neuropharmacol. 2016; 14(1):101-15.

4. Lanctôt KL, Herrmann N, Yau KK, Khan LR, Liu BA, LouLou $\mathrm{MM}$, et al. Efficacy and safety of cholinesterase inhibitors in Alzheimer's disease: a meta-analysis. CMAJ. 2003; 169(6):557-64.

5. Whitehead A, Perdomo C, Pratt RD, Birks J, Wilcock GK, Evans JG. Donepezil for the symptomatic treatment of patients with mild to moderate Alzheimer's disease: A meta-analysis of individual patient data from randomised controlled trials. Int J Geriatr Psychiatry. 2004; 19(7):624-33.

6. Farlow MR, Salloway S, Tariot PN, Yardley J, Moline ML, Wang

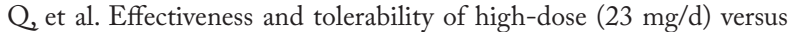
standard-dose $(10 \mathrm{mg} / \mathrm{d})$ donepezil in moderate to severe Alzheimer's disease: A 24-week, randomized, double-blind study. Clin Ther. 2010; 32(7):1234-51.

7. Cummings JL, Geldmacher D, Farlow M, Sabbagh M, Christensen D, Betz P. High-dose Donepezil $(23 \mathrm{mg} /$ day $)$ for the treatment of moderate and severe Alzheimer's Disease: drug profile and clinical guidelines. CNS Neurosci Ther. 2013;19(5):294-301.
8. Doody RS, Corey-Bloom J, Zhang R, Li H, Ieni J, Schindler R Safety and tolerability of donepezil at doses up to $20 \mathrm{mg} /$ day: results from a pilot study in patients with Alzheimer's disease. Drugs Aging. 2008; 25(2):163-74.

9. Higgins JPT, Green S. Cochrane handbook for systematic reviews of interventions Version 5.1.0. The Cochrane Collaboration. 2011.

10. Liberati A, Altman DG, TetzlaffJ, Mulrow C, Gøtzsche PC, Ioannidis JP, et al. The PRISMA Statement for reporting systematic reviews and meta-analyses of studies that evaluate health care interventions: explanation and elaboration. PloS Med. 2009;6(7):e1000100.

11. McKhann GM, Knopman DS, Chertkow H, Hyman BT, Jack CR Jr, Kawas CH, et al. The diagnosis of dementia due to Alzheimer's disease: recommendations from the national institute on agingAlzheimer's association workgroups on diagnostic guidelines for Alzheimer's disease. Alzheimers Dement. 2011; 7(3):263-9.

12. American Psychiatric Association. Diagnostic and Statistical Manual of Mental Disorders, Fifth Edition (DSM-5). 2013 American Psychiatric Association, Arlington, VA.

13. Homma A, Atarashi H, Kubota N, Nakai K, Takase T. Efficacy and safety of sustained release Donepezil high dose versus immediate release Donepezil standard dose in Japanese patients with severe Alzheimer's Disease: a randomized, double-blind trial. J Alzheimers Dis. 2016; 52(1):345-57.

14. Jackson S, Ham RJ, Wilkinson D. The safety and tolerability of donepezil in patients with Alzheimer's disease. Br J Clin Pharmacol. 2004; 58 Suppl 1:1-8.

15. Zanetti O, Solerte SB, Cantoni F. Life expectancy in Alzheimer's disease (AD). Arch Gerontol Geriatr. 2009; 49 Suppl 1:237-43. doi:10.1016/j.archger.2009.09.035.

16. Dumville JC, Hahn S, Miles JN, Torgerson DJ. The use of unequal randomisation ratios in clinical trials: a review. Contemp Clin Trials. 2006; 27(1):1-12.

17. Birks J, Harvey RJ. Donepezil for dementia due to Alzheimer's disease. Cochrane Database Syst Rev. 2006; (1):CD001190.

18. Molnar FJ, Hutton B, Fergusson D. Does analysis using "last observation carried forward" introduce bias in dementia research? CMAJ. 2008; 179(8):751-3. 